\title{
EL PAPEL DEL MAESTRO Y LA ESCUELA EN LA PROTECCIÓN INFANTIL. DETECCIÓN DE CASOS Y NOTIFICACIÓN EN LOS SERVICIOS DE PROTECCIÓN INFANTIL EN LA RIOJA
}

\author{
Alicia Pérez de Albéniz Iturriaga \\ Beatriz Lucas Molina \\ María Teresa Pascual Sufrate \\ Universidad de La Rioja
}

\begin{abstract}
RESUMEN: En la actualidad una de las cuestiones que presenta mayor interés en el ámbito profesional de la Protección Infantil es la necesidad de establecer sistemas de trabajo que posibiliten la coordinación entre los diferentes recursos e instituciones y entre las diferentes disciplinas que trabajan alrededor de la familia y la infancia. El sistema educativo forma parte imprescindible del sistema de recursos que trabajan con los niños y niñas víctimas de malos tratos. Sin embargo, son muchas las dificultades y reticencias que presentan los profesionales de la educación (maestros, profesores, personal directivo) para detectar estas situaciones e implicarse en su resolución. El presente artículo tiene como objetivo sensibilizar a estos profesionales con el triple objetivo de justificar su papel en el abordaje del problema, vencer algunas de las mencionadas dificultades e informar sobre el procedimiento de notificación en la Comunidad Autónoma de La Rioja.
\end{abstract}

ABSTRACT: Currently one of the issues most frequently discussed in the professional field of child protection is the need to establish working systems that enable coordination between the different resources and institutions and between different disciplines who work around the family and children. The education system is an essential part of system resources that work with victims of child abuse. But there are many difficulties and reluctance that present the education professionals (teachers, professors, staff) to detect these situations and get involved in their resolution. This article aims to raise awareness among professionals with the triple objective of justifying its role in addressing the problem, to overcome some of the above difficulties and report on the notification procedure in the Comunidad Autónoma de La Rioja.

PALABRAS CLAVE: Maltrato infantil, detección, notificación, servicios educativos.

KEYWORDS: Child abuse, detection, reporting, education system.

Recibido: $14 / 07 / 2010$

Aceptado: 15/10/2010 
En la actualidad existe consenso en buena parte de la sociedad occidental sobre la importancia de proteger a los niños y las niñas de todo tipo de situaciones de malos tratos de las que puedan ser víctimas. Las tasas de incidencia y prevalencia observadas en la mayoría de las investigaciones realizadas hasta la fecha y la constatación de las graves consecuencias que pueden conllevar estas situaciones para los niños y las niñas, confirman la gravedad del problema y señalan la urgencia de alcanzar un nivel adecuado de eficacia en la intervención social.

Sin embargo, las investigaciones sobre notificación de situaciones de maltrato infantil siguen indicando que los casos de infantes objeto de maltrato continúan llegando a los servicios de Protección Infantil mucho después de que estas situaciones hayan comenzado. La investigación también ha constatado que la cronicidad tiene una relación directa con la gravedad de los casos y un peor pronóstico (De Paúl y Arruabarrena, 1996; U.S. Department of Health and Human Services, Administration on Children, Youth and Families, 2007).

Una de las medidas que pueden asumirse para mejorar esta situación es implicar a todos los profesionales relacionados con la infancia (servicios educativos, sanitarios, de seguridad...). En este sentido, tal y como se argumentará con posterioridad en el presente artículo, la escuela es un observatorio privilegiado del desarrollo de nuestros niños y niñas, y supone una fuente inestimable de recursos para la detección, la notificación y la prevención (a todos los niveles) de las situaciones de maltrato/abandono infantil.

La Ley Orgánica de Educación 2/2006 establece en el Título II, en referencia al Principio de Equidad en la Educación, Capítulo I, una definición de alumnado con necesidad específica de apoyo educativo. En el artículo 71 de la citada ley se insta a las Administraciones a disponer de los medios necesarios para que todo el alumnado alcance el máximo desarrollo personal, intelectual, social y emocional. Se plantea, asimismo, una definición de necesidades educativas especiales en las que se incluyen, entre otras, las condiciones personales en las que se encuentren los niños que hagan que éstos requieran atención especial para lograr dicho desarrollo.

Sin embargo, son muchas las dificultades y reticencias que presentan los profesionales de la educación (maestros, profesores, personal directivo) para detectar estas situaciones e implicarse en su resolución. El presente artículo tiene como objetivo sensibilizar a estos profesionales con un triple propósito: 1) justificar el papel del educador en el abordaje del problema, 2) vencer dichas reticencias para la detección y notificación del maltrato y 3) informar sobre el procedimiento de notificación en la Comunidad Autónoma de La Rioja y aportar algunas indicaciones importantes sobre qué hacer y no hacer ante estas situaciones.

\section{¿Por Qué los Profesionales de la educación han de implicarse en el ABORDAJE DEL MALTRATO INFANTIL?}

Son muchas las razones por las que los educadores deben implicarse en las tareas de detección, notificación, tratamiento y prevención del maltrato infantil. En primer lugar, existe una obligatoriedad a nivel legal que es ineludible. Tal y como se ha planteado con anterioridad, la Ley Orgánica de Educación 2/2006 establece que las condiciones personales en las que se encuentran estos niños son objeto de especial 
atención. Además, la Ley Orgánica 1/1996, de 15 de enero, de Protección Jurídica del Menor, en su Título II, Capítulo I, Artículo 13, establece que Toda persona o autoridad y, especialmente, aquellos que por su profesión o función detecten una situación de riesgo o posible desamparo de un menor, lo comunicarán a la autoridad o sus agentes más próximos, sin perjuicio de prestarle el auxilio inmediato que precise.

En segundo lugar, la razón más práctica es que este fenómeno forma parte de la realidad cotidiana con y para los niños. Cada educador se enfrentará, con toda probabilidad, a lo largo de su carrera, con niños y niñas objeto de maltrato y estará en una posición aventajada para ayudar a estos niños y sus familias, así como a los servicios responsables de su tratamiento.

Además, los educadores deben prestar especial atención al problema porque la investigación parece indicar que muchos de los casos detectados se encuentran en edad escolar (Cawson, Wattam, Brooker y Kelly, 2000; Saldaña, Jiménez y Oliva, 1995). A menudo, puede que el maltrato haya comenzado antes de llegar a esta edad, pero estas situaciones no llegan a ser detectadas por nadie hasta que el niño entra en la escuela.

En cuarto lugar, la escuela es el único espacio donde los niños son atendidos diariamente durante largos periodos de tiempo por profesionales formados para observar su apariencia y conducta. Esto es especialmente relevante en un contexto como el nuestro, en el que se consideran las instituciones educativas como un recurso para cubrir muchas de las necesidades infantiles, no solo las referentes al nivel cognitivo.

En quinta instancia, el maltrato infantil se encuentra directamente relacionado con el aprendizaje. Tal y como se presentará posteriormente, la literatura muestra que estos niños manifiestan graves problemas a nivel académico (Cantón y CantónCortés, 2007; Cicchetti y Toth, 2005). Según lo establecido por la LOE 2/2006, los profesores no pueden ignorar aquellas condiciones personales que están impidiendo su desarrollo a este nivel.

Por último, a nivel ético, un educador debe comprometerse con este problema. Existe una responsabilidad compartida por todos para evitar o mitigar los efectos de este problema social y la posición en la que se encuentran los educadores les obliga a adoptar un papel activo ante estos casos.

\section{Principales dificultades Para la Detección del maltrato infantil}

Entre las dificultades que hacen referencia a la detección de situaciones de desprotección infantil, se encuentran algunas características propias del problema (De Paúl y Arruabarrena, 1996) como, por ejemplo, la habitual incapacidad por parte de los miembros de la familia para pedir ayuda, la invisibilidad de las lesiones del maltrato en muchos casos o el hecho de que los actos de maltrato ocurran en la intimidad familiar y sin testigos, de manera que no puedan observarse directamente. Tal y como establecen Staller y Faller (2010), el maltrato infantil es uno de los crímenes más difíciles de detectar y perseguir, principalmente porque a menudo no hay otro testigo que la propia víctima.

Pero, además, existen otro tipo de dificultades que afectan especialmente a los profesionales de la educación y que se presentan a continuación. 
Por un lado, existe un desconocimiento por parte de los profesionales de cuáles son las situaciones de desprotección infantil y sus indicadores. La primera aportación de los profesionales de la educación se basa en su capacidad para reconocer la existencia de niños o niñas que sean víctimas de malos tratos por parte de sus padres. Ser capaz de reconocer su existencia implica la detección de un posible caso de menor maltratado a partir de la presencia de una serie de indicadores de tipo físico o psicológico en el niño o en los padres. Es muy importante, por tanto, que los profesionales dispongan de la sensibilidad y la formación adecuadas para detectar este tipo de situaciones. Los indicadores de maltrato en los niños y en los padres que son observables por los educadores pueden ser bastantes inespecíficos, es decir, su presencia no garantiza que se trate de un caso de maltrato. Por consiguiente, la sensibilidad hacia este tipo de situaciones debe ir acompañada de la correspondiente formación que permita discriminar aquellas situaciones en las que los indicios son suficientemente razonables y precisos de aquéllas en las que no se da esta circunstancia (De Paúl y Perez de Albéniz, 2007).

Asimismo, existe entre los profesionales un desconocimiento de las consecuencias del maltrato infantil en los niños y niñas víctimas. Lejos de pretender una exposición exhaustiva sobre las consecuencias, sí se persigue mostrar que las situaciones de maltrato revelan gravísimas disfunciones relacionales que afectan al normal cumplimiento de las tareas evolutivas del niño (ver Cicchetti y Toth, 2003, para revisión). Ahora bien, el impacto de los negativos efectos del maltrato y el curso que éstos siguen en el niño no son, en modo alguno, lineales. Las consecuencias del maltrato representan un fenómeno cuya complejidad queda ilustrada cuando se observa que unas víctimas generan unos problemas y no otros, que éstos problemas pueden agravarse o bien remitir con el tiempo, que se manifiestan tardíamente o, incluso, que haya víctimas asintomáticas y ajustadas (Fergusson y Horwood, 2003). De hecho, el impacto del maltrato puede verse potenciado o amortiguado por múltiples variables como el momento evolutivo del niño, la tipología de maltrato, su gravedad, su cronicidad, su frecuencia, las características del perpetrador, del niño o del contexto extra-familiar y comunitario, entre otras.

No obstante, la literatura muestra que las situaciones de maltrato y abandono en los niños dificultan su adecuado desarrollo. En primer lugar, se han descrito diferentes consecuencias a nivel físico que pueden variar desde la muerte de los niños (Sanders, Colton y Roberts, 1999) hasta diferentes tipos de lesiones cutáneas, bucales, genitales, óseas o internas, como los traumatismos craneales y oculares que pueden condicionar retrasos, déficit motores o cognitivos y un sin fin de efectos a nivel físico (Martínez-Roig y De Paúl, 1993). En segundo lugar, la investigación sobre el maltrato en edades escolares ha constatado en reiteradas ocasiones que los niños que padecen malos tratos presentan un funcionamiento comportamental problemático mostrado mediante conductas de agresividad verbal y física, hostilidad, oposición, robos, mentiras y/o absentismo, que se integran en la categoría de "problemas de conducta" o externalizantes. Aunque estos problemas sean los más frecuentes, la faceta internalizante (Toth, Manly y Cicchetti, 1992) e incluso la combinación de ambas, también se ha encontrado en estos niños (De Paúl y Arruabarrena, 1996). En tercer lugar, los niños maltratados también muestran carencias emocionales y un funcionamiento pobre a nivel de salud mental (Armenta y Romero, 2008; Banyard, 
Williams y Siegel, 2001; Brown y Anderson, 1991; Myers, Berliner, Briere, Hendrix, Jenny y Reid, 2002). Por ejemplo, además de presentar mayor probabilidad de establecer un vínculo de apego inseguro (Cantón y Cortés, 1997; Crittenden, 1992; Crittenden y Ainsworth, 1989), los hallazgos de los estudios apuntan a una mayor presencia de sintomatología depresiva en escolares con problemas de maltrato (Cerezo y Frias, 1994; Fantuzzo, 1990; Kazdin, Moser, Colbus y Bell, 1985).

Respecto a las consecuencias que el maltrato puede provocar sobre la cognición infantil, los estudios coinciden en indicar que los niños maltratados tienen dificultades para etiquetar sentimientos, percibir intenciones en los demás y comprender roles sociales complejos (Straker y Jacobson, 1981). Además, se ha observado que obtienen peores resultados en el ámbito académico y presentan mayores problemas en esta área (Cantón y Cortés, 1997; Cicchetti y Toth, 2005; Shonk y Cicchetti, 2001; Stone, 2007) y que presentan dificultades de interacción social (Cerezo y Frías, 1994; Hofman-Plotkin y Twentyman, 1984; Klimes-Dougan y Kistner, 1990; Main y George, 1985). Todos estos datos, entre otros, dejan patente la necesidad de movilizar a todos los profesionales implicados en el trabajo con la infancia.

Además, los profesionales pueden realizar atribuciones erróneas sobre las causas del maltrato infantil. No hay una causa única del maltrato infantil, los padres que abusan de sus hijos son personas normales que se encuentran en situaciones sobre las que no tienen control. El hecho de que resulte difícil de entender que un padre pueda hacer daño a su propio hijo, si no es por presentar un grave trastorno mental, hizo que los investigadores dirigieran sus esfuerzos a investigar esta cuestión. Los primeros modelos planteados fueron los modelos psiquiátricos provenientes, principalmente, de la perspectiva psicoanalítica (Zigler y Hall, 1989). Estos modelos planteaban que los/las padres/madres maltratantes eran enfermos mentales, psicóticos y sádicos, así como que tenían trastornos de personalidad (Kaufman, 1962; Melnick y Hurley, 1969; Spinetta y Rigler, 1972). La explicación de la conducta maltratante, a través de la existencia de trastornos mentales, sin embargo, sería rechazada tras una década de investigación (Wolfe, 1987). La compilación de evidencia empírica mostró que sólo entre el 5 y el 10 por ciento de los/las padres/madres maltratantes eran diagnosticados con un trastorno psiquiátrico específico (Kelly, 1983). Asimismo, la investigación no mostró un perfil de personalidad específico que caracterizara a los maltratadores (Ammerman, 1990). El maltrato infantil es un problema que no distingue entre niveles económicos, creencias religiosas, razas, etc., aunque es cierto que existen algunos grupos que parecen ser más vulnerables debido a sus condiciones de vida, a sus características o a sus valores. La mayoría de los padres que maltratan a sus hijos son personas que quieren a sus hijos, pero que están pasando por situaciones que están fuera de su control. Los servicios de Protección Infantil pretenden, y consiguen en muchos casos, ofrecer a los niños y sus familias los recursos necesarios para poder afrontar estas situaciones y superarlas, para poder así hacerse cargo de los niños en un entorno adecuado de seguridad.

Por otra parte, muchos profesionales piensan que el problema es muy poco frecuente y que es poco probable que puedan encontrarse con casos de maltrato en su contexto. Lejos de ser un problema aislado de escasa incidencia y prevalencia, el maltrato infantil sigue siendo hoy un problema social que afecta a muchos niños y niñas de todas las culturas. Por ejemplo, el informe publicado por el Department of 
Health and Human Services de Estados Unidos (U.S. DHHS, 2007) muestra que un total de 899.000 casos de maltrato infantil fueron confirmados en el año 2005 (lo que supone el 1,2\% de la población infantil).

El único estudio a nivel nacional Ilevado a cabo hasta la fecha lo realizaron Saldaña, Jiménez y Oliva (1995) sobre expedientes de protección abiertos durante los años 1992 y 1993. Los resultados indicaron que la incidencia fue del 0,44 por mil en esos años. Las diferencias en las tasas observadas no responden más que a diferencias a nivel metodológico entre los estudios. Por ejemplo, el estudio español utilizó exclusivamente expedientes de los servicios de Protección Infantil y, por tanto, las tasas aparecen muy bajas. En cuanto a investigaciones sobre tipologías concretas, Pereda, Guilera, Forns y Gómez-Benito (2009) realizaron un meta-análisis para conocer la prevalencia del abuso sexual infantil con 65 trabajos de investigación de 22 países diferentes. Los resultados indicaron que el 7,4\% de los hombres y el $19,2 \%$ de las mujeres habían sufrido alguna forma de abuso sexual antes de cumplir los 18 años.

Una de las principales conclusiones cuando se analiza esta cuestión es que lo que se conoce en los servicios de Protección Infantil y a través de los estudios es simplemente "la punta del iceberg" que deja por debajo una gran cantidad de casos que no llegan a ser conocidos y que se ha denominado la "cifra negra" (Morales y Costa, 1997).

Asimismo, los profesionales pueden realizar atribuciones erróneas de la desprotección a costumbres y prácticas culturales. A menudo, los profesionales de la educación no actúan porque consideran que los valores de las familias con las que trabajan van a impedir un cambio. Es necesario ser conscientes de que todos los niños tienen una serie de necesidades básicas que han de ser cubiertas, independientemente de los valores culturales de sus familias. Las leyes que definen lo que es la Protección Infantil son aplicables a todas las familias de esa comunidad, sin excepciones culturales.

Además, los profesionales pueden pensar que no tienen derecho a inmiscuirse en la manera en la que los padres educan y cuidan a sus hijos. Es necesario reconocer que los niños no son una propiedad privada de los padres. El hecho de ser padre no significa poder tratar a un niño de la manera en que uno desee. En los casos de maltrato los padres hacen daño a sus hijos. Nadie puede tolerar que eso ocurra.

Finalmente, muchos profesionales intentan evitar la responsabilidad directa de intervenir en las situaciones de maltrato. Como ya ha sido planteado con anterioridad, la responsabilidad de garantizar el bienestar de los niños es de todos los ciudadanos y del conjunto de la sociedad. Es necesaria la colaboración de todos para identificar a los niños objeto de malos tratos, especialmente las personas que trabajan de manera directa con la infancia. Sin su apoyo los casos no llegarían nunca a los servicios de Protección Infantil.

\section{Principales dificultades PARA la NOtificaCiÓN DEl MALTRATO INFANTIL}

En cuanto a las dificultades para la notificación de situaciones de maltrato infantil el panorama es similar. Existen impedimentos por parte de los profesionales que 
deben ser rebatidos si se pretenden que los casos que sean detectados Ileguen a ser puestos en conocimiento de las autoridades competentes.

Por un lado, la experiencia de notificaciones anteriores en las que el educador puede considerar que no ha habido una respuesta satisfactoria por parte de los servicios de Protección Infantil, puede crear una expectativa de inutilidad de poner en conocimiento de estos servicios los nuevos casos detectados. La respuesta de los servicios de Protección Infantil va a ser diferente en cada caso en función de sus características. Confiar en la profesionalidad de las personas responsables de la intervención en los casos va a ser una condición indispensable. El informante debe saber que puede no recibir información posterior sobre la intervención llevada a cabo, no porque no haya habido una respuesta sino porque los servicios persiguen proteger el derecho a la intimidad de las familias.

Además, existe en algunos profesionales una incredulidad en que se pueda hacer algo para resolver la situación de la familia. Aunque es difícil determinar el pronóstico familiar en muchos casos, el hecho de no notificar mantiene al niño en una situación de riesgo y se impide que los servicios de Protección Infantil dispongan los recursos necesarios para intentar rehabilitar a la familia o mitigar las consecuencias de la situación de desprotección.

Por otro lado, un impedimento muy habitual entre los profesionales suele ser el miedo a que la sospecha no resulte cierta o no sea posible verificarla. Es muy importante que los profesionales, en general, sean conscientes de que no son los responsables de determinar si una situación es o no maltrato, sino los profesionales de los servicios de Protección Infantil. Además, en caso de temores sobre posibles represalias a nivel judicial de los padres, los servicios de Protección Infantil deben proteger y ayudar legalmente al informante. Otro temor suelen ser las posibles agresiones de los padres al conocer la notificación. La mayoría de los padres maltratantes no se enfrentan ni agreden directamente a otros adultos. Además, existe la posibilidad de realizar notificaciones anónimas en aquellos casos en los que se prevea su necesidad. Aunque el anonimato no suele ser aconsejable para evitar notificaciones realizadas con mala fe y porque el informante puede ser necesario con posterioridad para recabar datos de interés en la investigación del caso, en algunos casos puede aceptarse.

Asimismo, los profesionales pueden temer que la notificación genere problemas con los compañeros de trabajo o les lleve a ser criticados por éstos. La idea que debe guiar las actuaciones de los profesionales debe ser que su implicación en los casos puede prevenir que un niño llegue a ser gravemente dañado.

Por último, y quizás más importante, puede que los profesionales se encuentren inseguros o desconozcan dónde y cómo deben realizar la notificación. Es muy importante que los servicios de Protección Infantil proporcionen a la población general y a los profesionales en contacto con la familia y la infancia información clara sobre los objetivos y funcionamiento de sus servicios. Uno de los objetivos del presente artículo consiste, precisamente, en dar a conocer a los profesionales de la educación el procedimiento para la notificación de casos en La Rioja. Este objetivo se desarrollará en el siguiente apartado. 


\section{Procedimiento de notificación en la Comunidad Autónoma DE LA RIOJA}

La Consejería de Servicios Sociales del Gobierno de La Rioja puso en marcha en el año 2006 el Programa de detección, notificación y registro de maltrato infantil. El Programa nace de la iniciativa de la Consejería de Servicios Sociales para conseguir la máxima efectividad en la protección integral del menor, la prevención de los riesgos, así como la defensa y la garantía de sus derechos. Este programa se realizó en colaboración con el Ministerio de Trabajo y Asuntos Sociales y el Observatorio de la Infancia. Dicho programa tenía como objetivos sensibilizar y motivar a los profesionales sobre su papel en la prevención y la detección del maltrato infantil, además de facilitar los procesos de detección y notificación de los casos de maltrato infantil por parte de los profesionales de cada sector, promoviendo su colaboración en el programa. Asimismo, el programa perseguía cuantificar y conocer la dimensión del problema del maltrato infantil en La Rioja.

Los destinatarios del plan son aquellos profesionales en contacto directo con la población infantil, pertenecientes a los diferentes ámbitos de Servicios Sociales, Cuerpos y Fuerzas de Seguridad, Sanitario y Escolar.

La Consejería diseñó y editó para cada ámbito concreto una serie de Guías informativas como instrumento que proporcionara pautas concretas de actuación para los diferentes profesionales en la detección, notificación y registro de maltrato infantil. Igualmente, la Consejería diseñó unas Hojas de notificación que son una serie de protocolos simples y de fácil cumplimentación, como herramienta que permite la comunicación de casos de una forma estandarizada.

En concreto, la Consejería editó para los servicios educativos una Guía (Detección, notificación y registro de casos de maltrato infantil desde los servicios educativos) que incluye información pormenorizada sobre diferentes cuestiones. En primer lugar, se realiza una exposición sobre la definición de las diferentes tipologías de maltrato infantil y su frecuencia. En segundo lugar, se describen las causas principales que provocan las situaciones de maltrato. En tercer lugar, se plantea el marco normativo y las actuaciones y medidas de protección existentes. En cuarto lugar, se expone la teoría de las necesidades infantiles y los indicadores que permiten la detección de las situaciones de maltrato. En quinto lugar, se explican los diferentes recursos y actuaciones para la intervención en situaciones de desprotección infantil y el papel que tienen los profesionales de la educación en el abordaje de dichas situaciones. Finalmente, se trabaja el tema de la notificación, incluyendo información sobre dificultades para realizarla, quién debe realizarla y dónde.

Asimismo, la Consejería desarrolló unos protocolos denominados Hojas de notificación de riesgo y maltrato infantil en el ámbito escolar que, como se ha planteado anteriormente, es una herramienta que permite la comunicación de casos de una forma estandarizada. En ellas se presentan una serie de indicadores de las principales tipologías de maltrato infantil (maltrato físico, negligencia, maltrato emocional y abuso sexual) que el informante debe señalar con diferentes niveles de gravedad (leve, moderado o grave). Se presenta, igualmente, una casilla para notificar situaciones de violencia entre iguales y un apartado de observaciones en el que el informante puede aportar la información que considere relevante y pertinente. En último lugar, se incorpora un apartado con datos sobre la identificación del menor y del notificador/informante. 
Como se ha comentado con anterioridad, una de las funciones específicas que pueden desarrollar los profesionales de la educación es la notificación de sospechas de maltrato infantil. El profesional de los servicios educativos no necesita realizar las correspondientes actuaciones de valoración que culminan en la confirmación o no de las sospechas ya que, tal y como hemos insistido a lo largo del presente documento, son los profesionales de los Servicios Sociales quienes tienen el deber y las herramientas necesarias para realizarlo.

Ante una sospecha razonable de que se está produciendo maltrato, el profesional de la educación debe informar el caso al servicio correspondiente. En España, los servicios de Protección Infantil están divididos en dos niveles. Por un lado, están los Servicios Sociales Comunitarios, que se encargan de los casos de Riesgo y los casos de maltrato de gravedad leve y moderada. Por otro lado, disponemos de los Servicios Sociales especializados, que se encargan de los casos de Desamparo y Riesgo de Desamparo.

Los Servicios Sociales Comunitarios o de Base dependen de los ayuntamientos y se puede decir que son la puerta de entrada a los servicios de Protección Infantil. En ellos se reciben los casos y se realiza una primera valoración de la gravedad y del riesgo en el que se encuentra el niño, y desde ellos se derivan los casos a los Servicios Especializados en los casos en los que se considere necesario.

En La Rioja, el procedimiento habitual desde los servicios educativos suele ser la canalización de los casos a través de los Equipos de Orientación Educativa y Psicopedagógica. Estos equipos tienen como principal objetivo el asesoramiento al profesorado, al alumnado y a las familias sobre aspectos relativos a las respuestas educativas adecuadas para la individualización de la enseñanza y la atención a la diversidad, la orientación académica y profesional, y la planificación de la función tutorial, entre otras. Los Orientadores en las etapas de Educación Infantil y Primaria trabajan en equipo y, generalmente, derivan los casos detectados al profesional de su equipo que cumple el rol de Profesor Técnico de Servicios a la Comunidad. Tal y como aparece en la Figura 1, el proceso de detección/notificación comienza cuando un maestro detecta el caso. Generalmente, éste pone en conocimiento del tutor del niño y de la Dirección del Centro sus sospechas. Posteriormente, se informa a la persona de referencia del Equipo de Orientación Educativa y Psicopedagógica, que será quien derive el caso a los Servicios Sociales.

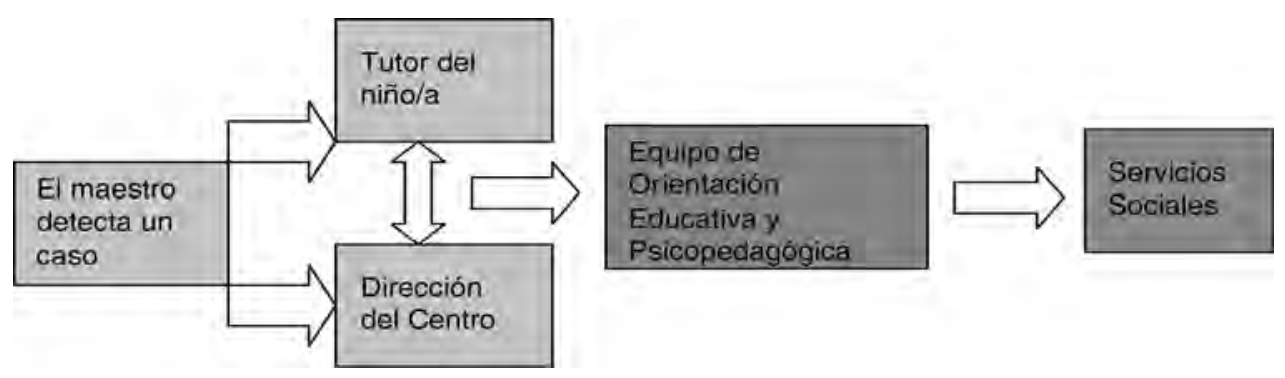

Figura 1. Proceso de notificación de casos de maltrato desde los servicios educativos 
Como puede apreciarse en la Figura 1, todo el proceso comienza con la detección por parte del maestro del caso. Sin este paso, el caso es muy poco probable que llegue a los Servicios Sociales. El maestro tiene a su disposición información valiosa sobre el estado físico, el comportamiento, el rendimiento académico y el estado psicológico/emocional del niño. Por otra parte, tiene información sobre el comportamiento y las actitudes de los padres y posee información acerca de la situación general de la familia. Estos datos pueden Ilevarle a sospechar la existencia de una situación de maltrato. Aunque son los Servicios Sociales los responsables de la investigación y la evaluación del caso, si el profesional de los servicios educativos decide realizar una entrevista con los padres, ésta debe producirse bajo las condiciones de máximo respeto, honestidad y profesionalidad. En ningún caso debe manifestar cólera o desaprobación hacia los padres ni juzgarles o amenazarles. Dado el alto nivel de formación requerido para afrontar estas situaciones, es preferible que las entrevistas se Ileven a cabo desde los Servicios Sociales. De manera similar, un primer impulso por parte de los profesionales de la educación suele ser el querer entrevistar al niño. A no ser que sea el propio niño el que se dirija directamente a él para revelar información sobre la situación de maltrato, lo deseable es que el profesional encargado de la investigación desde los Servicios Sociales junto al maestro, determine quién lleve a cabo la entrevista, cómo y cuándo.

De esta manera, el profesional de los servicios educativos no necesita realizar las correspondientes actuaciones de valoración que culminan en la confirmación o no de las sospechas ya que, tal y como hemos insistido a lo largo del presente documento, son los profesionales de los Servicios Sociales quienes tienen el deber y las herramientas necesarias para hacerlo. Ante una sospecha razonable de que se está produciendo maltrato, el profesional de la educación sólo debe informar del caso a través de los cauces que se han señalado.

Una excepción a lo expuesto hasta el momento son las situaciones de emergencia en las que el profesional de la educación puede percibir que la integridad física y mental de un niño, incluso su vida, se encuentran en grave peligro. En estos supuestos se debe contactar de manera inmediata con la Consejería de Servicios Sociales del Gobierno de La Rioja, cuyos profesionales decidirán qué decisiones y medidas se han de tomar. Sin embargo, si las condiciones no permiten dicho contacto inmediato, se debe acudir igualmente de manera urgente al Juzgado de Guardia o al Ministerio Fiscal.

Una vez iniciado el proceso, el profesional de la educación podrá ser requerido para colaborar con los servicios de Protección Infantil en las tareas de investigación e intervención. Por un lado, podrá aportar información que posea sobre el menor y la situación familiar en el proceso de valoración del caso por parte de los profesionales de los Servicios Sociales. Esta colaboración permitirá disponer de hipótesis más validas sobre las causas y los factores de riesgo que están provocando y manteniendo la posible situación de maltrato. Por otro lado podrá colaborar, junto con otros servicios (salud mental, Servicios Sociales, etc.) en el abordaje de las consecuencias y correlatos (hiperactividad, síntomas depresivos, agresividad, necesidades afectivas no cubiertas, etc.) de los malos tratos en los niños, gracias a su formación profesional y al contacto diario y la relación de confianza con el niño. 
En La Rioja, durante el año 2009, fueron un total de 45 las notificaciones de maltrato o sospecha de maltrato recibidas en la Consejería de Servicios Sociales. De ellas, un 46,6\% fueron realizadas desde el ámbito de los propios Servicios Sociales, un $37,7 \%$ fueron realizadas desde el ámbito escolar, el 15,5\% desde el ámbito policial y ninguna desde los servicios de salud. Sería necesario comparar estas cifras con aquéllas registradas con anterioridad al planteamiento del Programa de detección, notificación y registro de maltrato infantil por parte de la Consejería para conocer su impacto e investigar qué está ocurriendo en los servicios sanitarios para que no notifiquen las situaciones de maltrato que probablemente detecten.

En cualquier caso, de los datos se deduce que los servicios educativos en la Comunidad Autónoma de La Rioja toman parte activa en la detección y notificación del maltrato infantil. Lo que habría que investigar es la proporción de casos que notifican y si los profesionales son sensibles a todas las tipologías de maltrato, ya que el $73,3 \%$ de los casos notificados por todos los profesionales incluían al menos la tipología de negligencia.

\section{CONCLUSiOnes}

Tal y como se ha mostrado en el presente artículo, el maltrato infantil es un problema social que puede conllevar serias consecuencias para los niños. Su incidencia, lejos de ser aislada, es muy alta y afecta a todo tipo de niños y niñas.

Existen, según se ha puesto de manifiesto, muchas otras razones para involucrarse en su resolución. Desde el punto de vista del profesional de la educación son dos las cuestiones que éste debería tener, además, en cuenta. En primer lugar, el hecho de que los casos Ileguen a los servicios de Protección Infantil de manera temprana puede hacer que dichas consecuencias no se produzcan o, si lo hacen, sea con menos intensidad. En segundo lugar, la literatura muestra que la figura del maestro o profesor puede tener un efecto protector en estas situaciones. Un factor protector es una variable que tiene un poder amortiguador y que modifica los efectos del riesgo en una dirección positiva (Luthar y Cicchetti, 2000). La investigación ha mostrado que los factores protectores a diferentes niveles (individual, familiar o comunitario) tienen un gran peso en la explicación de por qué algunos niños sufren determinadas consecuencias derivadas del maltrato y otros no. A nivel individual, las características del niño como sus habilidades sociales, su capacidad de resolución de problemas, la autonomía y la autoestima positiva, entre otros, se han mostrado factores protectores contra el impacto negativo del maltrato (Bernard, 1991; Luthar y Zigler, 1991). Características familiares, como el apoyo familiar, pueden funcionar, asimismo, como amortiguadores del impacto del maltrato (Overstreet, Dempsey, Graham y Moely, 1999).

Pero lo más relevante para el tema que nos ocupa es que la investigación muestra que los niños pueden recibir este apoyo desde fuera de la familia y que los maestros pueden ser esa figura que les aporta el soporte necesario para atenuar las consecuencias del maltrato u otras adversidades (Rak y Patterson, 1996).

Además, consideramos importante insistir en que los profesionales en general deben ser conscientes de que no son los responsables de determinar si una situación es o no maltrato ni de establecer los recursos para su resolución. Son los profesiona- 
les de los servicios de Protección Infantil los responsables de asumir la tarea de investigar y establecer una intervención en caso necesario. Sin embargo, sin asumir la responsabilidad que conlleva la detección y notificación de los casos de maltrato infantil por parte de los profesionales de la educación, esos niños difícilmente recibirán la ayuda que necesitan y continuarán formando parte de la citada "cifra negra".

Los maestros son, frecuentemente, la primera figura de autoridad con la que se relacionan los niños fuera de sus hogares. Por tanto, pueden jugar un rol en su protección y ayudar al niño que identifican con riesgo o que les realiza una revelación (Rossato y Brackenridge, 2009).

Sin embargo, la investigación muestra que la situación actual dista mucho de ser la idónea. Por ejemplo, el estudio Ilevado a cabo por Baginsky y Hodgkinson (1999) en Inglaterra y Gales encontró que la formación en protección infantil para los maestros era infrecuente y que los departamentos universitarios encargados de la instrucción de maestros carecían de consistencia en la cantidad de tiempo y contenidos dedicados a la formación sobre protección infantil. En la actualidad, el tema de maltrato infantil se encuentra en muchos de los planes de estudio de nuestro entorno, pero sería necesario realizar estudios con el fin de determinar si esta formación está siendo suficiente y adecuada.

\section{REFERENCIAS BIBLIOGRÁFICAS}

AMMERMAN, R. T. (1990). Etiological models of child maltreatment. A behavioral perspective. Behavior Modification, 14, 230-254.

ARMENTA, M. y ROMERO, J. (2008). Consecuencias de la violencia familiar experimentada directa e indirectamente en niños: Depresión, ansiedad, conducta antisocial y ejecución académica. Revista Mexicana de Psicología, 2, 237-248.

BAGINSKY, M. y HODGKINSON, K. (1999). Child protection training in initial teacher training: A survey of provision in institutions of higher education. Educational Research Volume, 41, 173-181.

BANYARD, V. L., WILLIAMS L. M. y SIEGEL, J. A. (2001). The long-term mental health consequences of child sexual abuse: An exploratory study of the impact of multiple traumas in a sample of women. Journal of Traumatic Stress, 14, 697-715.

BERNARD, B. (1991). Fostering resiliency in kids: Protective factors in the family, school and community. Portland, OR: Western Regional Center for Drug-Free Schools and Comunities.

BROWN, G. R. y ANDERSON, B. (1991). Psychiatric morbidity in adult inpatients with childhood histories of sexual and physical abuse. American Journal of Psychiatry, 148, 55-61.

CANTÓN, J. y CORTÉS, M. R. (1997). Malos tratos y abuso sexual infantil. México: Siglo XXI.

CANTÓN, J. y CANTÓN-CORTÉS, D. (2007). Abuso sexual infantil. En E. Fernández de Haro, F. Justicia, M.C. Pichardo (Eds.), Enciclopedia de psicología evolutiva y de la educación (pp. 401-422). Madrid: Aljibe. 
CAWSON, P., WATTAM, C., BROOKER, S. y KELLY, G. (2000). Child Maltreatment in the UK: A study of the prevalence of child abuse and neglect. London: National Society for Prevention of Cruelty to Children.

CEREZO, M. A. y FRIAS, D. (1994). Emotional and cognitive adjustment in abused children. Child Abuse \& Neglect, 18, 923-932.

CICCHETTI, D. y TOTH, S. (2003). Child maltreatment: Past, present, and future perspectives. En R. Weissberg, H. Walberg, M. O’Brien y C. Kuster, (Eds.), Longterm trends in the well-being of children and youth: Issues in children's and families lives (pp. 181-205). Child Welfare League of America, Washington, DC.

CICCHETTI, D. y TOTH, S. (2005). Child maltreatment, Annual Review of Clinical Psychology 1, 409-438.

CRITTENDEN, P. (1992). Children's strategies for coping with adverse home environments: An interpretation using attachment theory. Child Abuse \& Neglect, 16, 329-343.

CRITTENDEN, P. y AINSWORTH, M. (1989). Child maltreatment and attachment theory. En D. Cicchetti y V. Carlson (Eds.), Child maltreatment: theory and research on the causes and consequences of child abuse and neglect (pp. 432463). New York: Cambridge University Press.

DE PAÚL, J. y ARRUABARRENA, M. I. (1996). Manual de Protección Infantil. Barcelona: Masson.

DE PAÚL, J. y PÉREZ DE ALBÉNIZ, A. (2007). Familias en las que hay malos tratos. En F. López (Coord.), La escuela infantil: observatorio privilegiado de las desigualdades, (pp. 137-154). Barcelona: Graó.

FANTUZZO, J. W. (1990). Behavioral treatment of the victims of child abuse and neglect. Child Abuse \& Neglect, 14, 316-339.

FERGUSSON, D. M. y HORWOOD, L. J. (2003). Resilience to childhood adversity: Results of a 21-year study. En S.S. Luthar (Ed.), Resiliency and vulnerability: Adaptation in the context of childhood adversities (pp. 130-155). Cambridge: Cambridge University Press.

GOBIERNO DE LA RIOJA. FAMILIA Y ACCIÓN SOCIAL (2003). Detección, notificación y registro de casos de maltrato infantil desde los servicios educativos.

GOBIERNO DE LA RIOJA. FAMILIA Y ACCIÓN SOCIAL. Hoja de notificación de riesgo y maltrato infantil en el ámbito escolar.

HOFFMAN-PLOTKIN, D. y TWENTYMAN, C. T. (1984). A multimodal assessment of behavioral and cognitive deficits in abused and neglected preschoolers. Child Development, 55, 794-802.

KAUFMAN, M. (1962). Psychiatric implications of physical abuse of children. En V. De Francis (Ed.), Protecting the battered child (pp. 17-22). Denver, CO: American Human Association.

KAZDIN, A. E., MOSER, J., COLBUS, D. y BELL, R. (1985). Depressive symptoms among physically abused and psychiatrically disturbed children. Journal of Abnormal Psychology, 94, 298-307.

KELLY, J. A. (1983). Treating child abusive families: Intervention based on skills-training principles. New York: Plenum. 
KLIMES-DOUGAN, B. y KISTNER, J. (1990). Physically abused preschoolers' responses to peers' distress. Developmental Psychology, 26, 599-602.

Ley Orgánica 1/1996, de 15 de enero, de Protección Jurídica del Menor (BOE número 15, miércoles 17 de enero de 1996).

Ley Orgánica 2/2006, de 3 de mayo, de Educación (BOE número 106, jueves 4 de mayo de 2006).

LUTHAR, S. y CICCHETTI, D. (2000). The construct of resilience: Implications for interventions and social policies. Development and Psychopatholgy, 12, 857-885.

LUTHAR, S. y ZIGLER, E. (1991). Vulnerability and competente: A review of research on resilience in childhood. American Journal of Orthopsychiatry, 61, 6-22.

MAIN, M. y GEORGE, C. (1985). Responses of abused and disadvantaged toddlers to distress in agemates: A study in the day-care setting. Developmental Psychology, 21, 407-412.

MARTÍNEZ-ROIG, A. y DE PAÚL, J. (1993). Maltrato y abandono a la infancia. Barcelona: Martínez Roca.

MELNICK, B. y HURLEY, J. R. (1969). Distinctive personality attributes of child abusing mothers. Journal of Consulting and Clinical Psychology, 33, 346-749.

MORALES, J. M. y COSTA, M. (1997). Tendencias actuales en la investigación del maltrato infantil. En J. Casado, J. A. Díaz y C. Martínez (Eds.), Niños maltratados (pp. 309-323). Madrid: Díaz de Santos.

MYERS, J., BERLINER, L., BRIERE, J., HENDRIX, C., JENNY, C. y REID, T. (2002). The APSAC handbook on child maltreatment ( $2^{\underline{a}}$ ed.). Thousand Oaks, CA: Sage Publications, Inc.

OVERSTREET, S., DEMPSEY, M., GRAHAM, D. y MOELY, B. (1999). Availability of family support as a moderatos of exposure to community violence. Journal of Clinical Child Psychology, 28, 151-159.

PEREDA, N., GUILERA, G., FORNS, M. y GÓMEZ-BENITO, J. (2009). The prevalence of child sexual abuse in community and student samples: A meta-analysis. Clinical Psychology Review, 29, 328-338.

RAK, C. y PATERSON, L. (1996). Promoting resilience in at-risk children. Journal of Counseling \& Development, 74, 368-373.

ROSSATO, C. (2009). Child protection training in sportrelated degrees and initial teacher training for physical education: An audit. Child Abuse Review, 18, 81-93.

SALDAÑA, D., JIMÉNEZ, J. y OLIVA, A. (1995). El maltrato infantil en España: Un estudio a través de los expedientes de menores. Infancia y Aprendizaje, 71, 59-68.

SANDERS, R., COLTON, M. y ROBERTS, S. (1999). Child abuse fatalities and causes of extreme concern: Lessons from reviews. Child Abuse \& Neglect, 23, 257-268.

SHONK, S. M. y CICCHETTI, D. (2001). Maltreatment, competency deficits, and risk for academic and behavioral maladjustment, Developmental Psychology, 37, 3-17.

SPINETTA, J. J. y RIGLER, D. (1972). The child abusing parent: A psychological review. Psychological Bulletin, 77, 296-304.

STALLER, K. y FALLER, K. (2010). Child sexual abuse: Legal burdens and scientific methods. New York, NY, US: Columbia University Press. 
STONE, S. (2007). Child maltreatment, out-of-home placement and academic vulnerability: A fifteen-year review of evidence and future directions. Children and Youth Services Review, 29, 139-161.

STRAKER, G. y JACOBSON, R. S. (1981). Aggression, emotional maladjustment and empathy in the abused child. Development and Psychopathology, 17, 762-765.

TOTH, S. L., MANLY, J. y CICCHETTI, D. (1992). Child maltreatment and vulnerability to depression, Development and Psychopathology, 4, 97-112.

U.S. Department of Health and Human Services, Administration on Children, Youth and Families. (2007). Child Maltreatment 2005. Washington, DC: U.S. Government Printing Office.

WOLFE, D. A. (1987). Child abuse: Implications for child development and psychopathology. London: Sage.

ZIGLER, E. y HALL, N. W. (1989). Child abuse in America: Past, present and future. En D, Cicchetti y V. Carlson (Eds.), Child maltreatment: Theory and research on the causes and consequences of child abuse and neglect (pp. 38-75). Cambridge: Cambridge University Press. 